\title{
Design of a Theory-Based Yoga Intervention to Promote Cardiovascular Health in Older Adults
}

\author{
Jennifer L Barrows* and Julie Fleury \\ College of Nursing and Health Innovation, Arizona State University, USA
}

Submission: August 21, 2017; Published: October 16, 2017

*Corresponding author: Jennifer Barrows, College of Nursing and Health Innovation, Arizona State University, 550 N. 3rd St. Phoenix, AZ 85004 , USA, Tel: (310) 993-4355; Email: jlbarrow@asu.edu

\begin{abstract}
Cardiovascular disease (CVD) is the leading cause of death in older adults. Physical activity has been shown to reduce CVD risk, yet most older adults do not engage in adequate physical activity to promote cardiovascular health. Yoga has been shown to reduce the risk of CVD through improvement in blood pressure, body composition, glucose tolerance, and lipid profile. However, few yoga interventions have been guided by theory, which limits our understanding of the mechanisms by which yoga is expected to achieve favorable changes in cardiovascular health among older adults. The purpose of this paper is to describe a theory-based approach to the design of the Yoga for HEART (Health Empowerment and Realizing Transformation) intervention to enhance motivation for health behavior change and improve health outcomes in communitydwelling older adults at risk for CVD. This pilot randomized controlled trial (RCT) will provide information about the feasibility of conducting a future definitive RCT.

Twenty ( $N=20)$ sedentary older adults ( $\geq 50$ years) will be randomly assigned to the yoga intervention group or to an attention control group. Intervention group participants will attend group yoga sessions for 60 minutes, twice per week, for 12 weeks. Attention control group participants will receive mailed weekly newsletters. The groups will be compared pre-intervention (T1) and post-intervention (T2) on theoretical mediating variables, physical activity behavior, cardiovascular health outcomes (i.e., blood pressure and body composition), and health-related physical fitness (i.e., endurance, strength, balance, and flexibility). Feasibility will be assessed in terms of intervention acceptability, demand, and implementation.
\end{abstract}

Keywords: Yoga; Theory; Intervention; Cardiovascular health; Older adults; Wellness; Motivation

\section{Introduction}

Cardiovascular disease (CVD), a broad diagnostic category encompassing hypertension, atherosclerosis, heart disease, peripheral vascular disease, and stroke, is the leading cause of mortality in the United States and worldwide [1,2]. An estimated 92.1 million American adults ( $>1$ in 3 ) are living with CVD; direct and indirect costs of CVD, including health expenditures and lost productivity, are estimated to total more than US $\$ 316$ billion annually [1]. Older adults experience a disproportionate burden of CVD; approximately $69 \%$ of US adults aged 60 to 79 years, and $85 \%$ of those aged $\geq 80$ years, have one or more types of CVD [3]. As the older adult population is projected to increase substantially in the next several decades, the socioeconomic costs of CVD will continue to rise [4]. To promote cardiovascular health and reduce the risk of CVD, the American Heart Association (AHA) endorses key health factors and behaviors, including: (a) maintaining a healthy blood pressure, (b) maintaining a healthy lipid profile, (c) maintaining a healthy blood glucose level, (d) maintaining a healthy body mass index, (e) engaging in physical activity, (f) consuming a healthy diet, and (g) avoiding tobacco use [5]. Despite knowledge of the health factors and behaviors contributing to cardiovascular health, the challenge of promoting sustained lifestyle change to reduce CVD risk remains unmet. Less than $9 \%$ of older adults ( $\geq 75$ years of age) meet federal physical activity guidelines to promote cardiovascular and overall health [6]. As the population ages and the burden of CVD grows, there is a critical need for innovative approaches to cardiovascular health promotion among older adults.

Yoga has been acknowledged as an effective strategy to promote cardiovascular health in older adults [7]. Yoga offers a mind-body approach to health, integrating components of physical activity, breathing, and meditation to enhance physical, psychological, and spiritual well-being $[8,9]$. A recent meta- 
analytic review reported that yoga was equally effective in reducing CVD risk when compared to aerobic physical activities such as brisk walking or cycling [10]. Yoga may be comparable or superior to other types of physical activity in managing blood pressure [10-12], body weight [11,13], blood glucose $[12,13]$, lipid profile $[10,11,13]$, and psychosocial stress $[14,15]$. Mooventhan \& Nivethitha [16] conducted a comprehensive review of the effects of yoga on chronic conditions in older adults, concluding that yoga improves health outcomes in multiple domains, including physical (e.g., blood pressure, heart rate variability, and insulin sensitivity), mental (e.g., depression and anxiety), emotional (e.g., mood and stress), and social (e.g., life satisfaction). Chu \& colleagues [17] compared the effectiveness of lifestyle interventions targeting both individual CVD risk profile and 10-year CVD risk, reporting that yoga, through providing the largest improvement in blood pressure and lipid levels, promotes significant reduction in CVD risk.

While the benefits of yoga in promoting cardiovascular health in older adults have been supported, there has been limited attention to theory as a basis for intervention design and evaluation [7]. In a recent commentary addressing the public health implications of yoga research, Patwardhan [18] emphasized the need for theory as a robust framework guiding yoga interventions. Theory presents a systematic way of understanding the process of health behavior change, including initiation and maintenance [19]. Theory provides an understanding of the problem that the intervention targets, the nature of the intervention needed to address the problem, and the mechanisms underlying the anticipated improvement in outcomes [20]. Theoretical frameworks foster a systematic approach to intervention development and implementation that allow us to move beyond a simplistic, outcomes-focused approach, to examining the central processes underlying intervention effects [21]. Thus, theoretical frameworks guide the development of interventions that are more likely to permit strong causal inference and interpretable results [22]. A theorybased approach to yoga interventions is essential to develop and expand the knowledge base for research and clinical practice.

Despite the strengths of a theory-based approach to interventions aimed at changing health behavior, few reports have described the use of theory in the design, implementation, and evaluation of yoga interventions for older adults. Thus, the purpose of this paper is to describe the design of Yoga for HEART (Health Empowerment and Realizing Transformation), atheory-based yoga intervention to enhance motivation for physical activity and improve cardiovascular health in older adults. The Yoga for HEART intervention is guided by Wellness Motivation Theory (WMT) $[23,24]$ and operationalized in light of the principles and practices of Astanga yoga [25,26]. Wellness motivation is conceptualized as a process of empowering potential for health behavior change consistent with an individual's unique strengths, values, and goals $[23,24]$. In the Yoga for HEART intervention, WMT provides a robust framework fostering the process through which motivation for health behavior change is initiated and sustained over time.

\section{Specific Aims}

The primary aim of this pilot study is to examine the feasibility (i.e., acceptability, demand, and implementation) of Yoga for HEART, a 12-week yoga intervention to increase motivation for physical activity and improve health outcomes in sedentary older adults at risk for CVD. Secondary aims of this study are to evaluate the efficacy of the intervention in promoting (a) behavioral change processes (i.e., self-knowledge, motivation appraisal, and self-regulation); (b) social contextual resources (i.e., social support and community resources); (c) physical activity behavior (i.e., intensity, frequency, and duration); (d) cardiovascular health outcomes (i.e., blood pressure, body mass index, and waist-hip ratio); and (e) health-related fitness (i.e., strength, endurance, balance, and flexibility), as compared with an attention control group. A pilot study will provide important information about the feasibility of conducting a future definitive RCT of the Yoga for HEART intervention. The study protocol is reported in accordance with Consolidated Standards of Reporting Trials (CONSORT) guidelines [27-29].

\section{Methods}

\section{Design}

This study is a single-center, single-blind, parallelgroup, pilot RCT to evaluate the feasibility of a 12 -week yoga intervention aimed at improving cardiovascular health outcomes in a sample of $\mathrm{N}=20$ sedentary community-dwelling older adults ( $\geq 50$ years of age). Participants will be randomly assigned, with a 1:1 allocation ratio, to one of two groups: (a) intervention (Yoga for HEART), or (b) attention control (weekly newsletters). Individuals randomized to the intervention group will participate in 60-minute group yoga sessions, twice per week, for 12 weeks. Individuals randomized to the control group will receive mailed weekly newsletters on topics related to healthy aging. Social contextual resources, behavioral change processes, physical activity behavior, and cardiovascular health outcomes will be assessed at baseline (T1) and 12 weeks (T2), immediately following program implementation. All assessments and group yoga sessions will take place at a community-based yoga studio in Los Angeles, CA. The Institutional Review Board at Arizona State University approved the study protocol (\#00002929). Informed consent will be obtained from all study participants prior to their participation in any research activities.

\section{Theory}

Wellness Motivation Theory provides a framework for the design, implementation, and evaluation of the Yoga for HEART intervention, grounded in nursing science and acknowledging the complexity and contextual relevance of motivation for yoga practice and cardiovascular health $[23,24]$. Intervention approaches testing WMT have demonstrated efficacy in promoting physical activity and improving health outcomes 
among older adults [30,31]. Wellness Motivation Theory is congruent with yoga philosophy in that health behavior change is conceptualized as: (a) a dynamic process of intention formation and action leading to new and positive health patterns; (b) building on individual and community strengths and resources to promote health and wellness consistent with personal goals and values; and (c) shaped by the individual in mutual interaction with the environment $[23,24]$.

\section{Participants}

Eligible participants will be community-dwelling older adults with low levels of physical activity who meet the following inclusion criteria: (a) $\geq 50$ years of age; (b) sedentary $(<150$ minutes of moderate intensity physical activity per week); (c) able to ambulate without an assistive device; (d) cognitively intact; (e) English language literate; and (f) willing and able to provide informed consent and comply with study procedures. A convenience sample of 20 participants will be recruited for this study. Recruitment efforts will include advertising in local newspapers, distributing flyers, and giving presentations throughout the community in locations that serve older adults, including recreation centers, retirement communities, physician offices, pharmacies, grocers, and other merchants. Individuals who express interest in study participation will be invited to schedule a telephone screening appointment with the primary investigator (PI) to explain the details of the study and determine eligibility status.

Potential participants will be assessed for eligibility during telephone interviews using a screening form that includes information about the individual's age, health status, cognitive status, and readiness for physical activity. The Physical Activity Readiness Questionnaire (PAR-Q) will be used to assess risk in accordance with American College of Sports Medicine (ACSM) guidelines [32]. Participants $\geq 69$ years of age and/or those who respond affirmatively to any PAR-Q items will be asked to obtain physician clearance prior to study participation. The Stages of Change Questionnaire will be used to confirm sedentary behavior; individuals who currently meet federal physical activity guidelines by engaging in $\geq 150$ minutes of moderate intensity physical activity per week (i.e., classified as stage 4: active or stage 5: maintenance) will be excluded from study participation [33-35]. The telephone version of the Mini Mental Status Exam (tMMSE) will be used to assess cognitive status; a score $\geq 21 / 26$ on the tMMSE, indicating no severe cognitive impairment such as dementia, will be required to provide informed consent and participate in the study [36].

Individuals who are eligible to participate will be invited to an enrollment appointment that combines informed consent and baseline testing procedures. Individuals who express an interest in yoga or physical activity, but do not meet inclusion criteria, will be referred to other community resources. Informed consent, data collection appointments, and group yoga sessions will take place at a community-based yoga studio in Los Angeles, CA.

\section{Yoga for HEART Intervention}

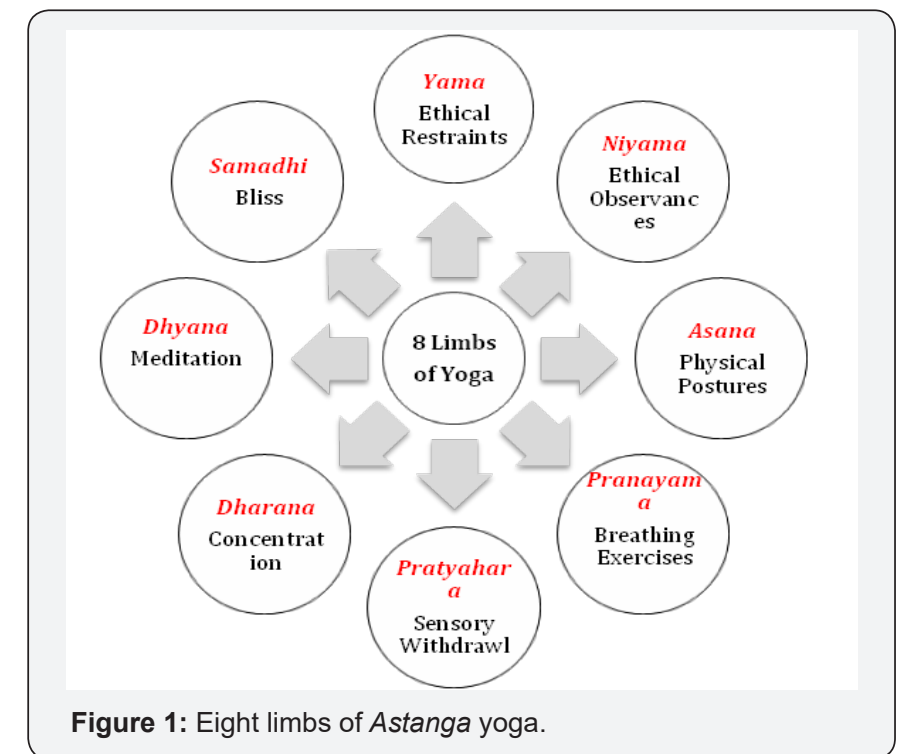

While there are many approaches to yoga practice, each with a different emphasis on the mind, body, and spirit, at its fundamental core, yoga is an ancient wisdom tradition, a philosophical framework for living and being well [8,37]. The Yoga for HEART intervention is based on principles of Astanga yoga [26]. The Astanga system of yoga is comprised of eight limbs, including yama (ethical restraints), niyama (ethical observances), asana (physical postures), pranayama (breathing exercises), pratyahara (sensory withdrawal), dharana (concentration), dhyana (meditation), and samadhi (selfactualization or bliss). The first two limbs, yama and niyama, provide the foundation for all other limbs of yoga. The yama-s consist of five ethical restraints, including ahimsa (non-harm) and satya (truthfulness), that guide yoga practice and support harmonious relationships with others and the environment. The niyama-s are comprised of five ethical observances, including tapas (discipline) and svadhyaya (self-study) that underlie yoga practice and encourage caring behavior toward the self and others [25]. Each of these ethical principles is woven throughout the Yoga for HEART intervention. For example, each class will place an emphasis on being kind to oneself and others; practicing in a safe manner that does not cause harm; engaging in self-study to identify strengths, abilities, and resources; committing to the process of change with focus, persistence, and discipline; letting go of judgements, expectations, and attachments; and replacing negative, unhealthy patterns with positive, healthy patterns of cognition, emotion, and behavior. The eight limbs of Astanga yoga are illustrated in Figure 1.

The Yoga for HEART intervention was developed by the PI, a registered nurse and a certified yoga instructor who has experience teaching yoga to older adults. The length of the 
program, the frequency and duration of yoga sessions, and the critical inputs of the intervention were based on reviews of the literature $[7,11]$. Small group yoga sessions will be held for 60 minutes, twice per week, for 12 weeks. Each yoga session will be limited to 10 participants to provide individualized attention and to promote safety. The yoga instructor will use an intervention manual and follow a standardized protocol, but will address personally relevant goals and concerns for each participant.

Table 1: Standard yoga session format.

\begin{tabular}{|c|c|}
\hline Curriculum Elements & Time (Min) \\
\hline Opening or centering exercise & 5 \\
\hline Physical postures & 40 \\
\hline Closing exercise with relaxation/meditation & 5 \\
\hline Group discussion & 10 \\
\hline Total & 60 \\
\hline
\end{tabular}

Table 2: Yoga for HEART intervention postures.

\begin{tabular}{|c|c|}
\hline Posture & Asana \\
\hline Mountain pose & Tadasana \\
\hline Mountain w/arms extended & UrdhvaHastasana \\
\hline Mountain w/fingers interlaced & Baddhanguliasana \\
\hline Forward fold & Uttanasana \\
\hline Downward facing dog pose & AdhoMukhaSvanasana \\
\hline Warrior II pose & Virabhadrasana II \\
\hline Plank pose & ArdhaChaturangaDandasana \\
\hline Four-limbed staff pose & ChaturangaDandasana \\
\hline Tree pose & Vrksasana \\
\hline Staff pose & Dandasana \\
\hline Seated forward fold & Paschimottanasana \\
\hline Simple twisting pose & Bharadvajasana \\
\hline Knees to chest pose & Apanasana \\
\hline Reclining twist pose & JatharaParivartanasana \\
\hline Final relaxation pose & Savasana \\
\hline Crescent pose & Anjaneyasana \\
\hline Sphynx pose & SalambaBhujangasana \\
\hline Bent knee pose & JanuSirsasana \\
\hline Bridge pose & Setu Bandha Sarvangasana \\
\hline Triangle pose & Trikonasana \\
\hline Marichi's pose & Marichyasana \\
\hline Mountain w/cow face arms & TadasanaGomukhasana \\
\hline Chair pose & Utkatasana \\
\hline Locust pose & Salabhasana \\
\hline Bound angle pose & Baddhakonasana \\
\hline Side angle pose & Parsvakonasana \\
\hline
\end{tabular}

Each session will begin with an opening or centering exercise in a comfortable and relaxed position with attention focused on the breath (Table 1). After a brief introduction to yoga philosophy, participants will practice physical postures in a specific sequence designed to promote endurance, strength, flexibility, and balance (Table 2). Yoga props, including blocks, straps, blankets, bolsters, and chairs, will be provided to adapt postures to meet individual needs. The difficulty level of each posture will progress gradually over time as participants gain knowledge, skills, confidence, fitness, and ability. Each yoga session will end with restorative postures and a closing exercise such as guided meditation and/or breath control. Finally, time will be allocated for small group discussion to foster dialogue among participants and facilitate the delivery of theory-based educational components, including motivational strategies such as goal setting, self-monitoring, social support, and problem solving to overcome obstacles to health behavior change.

\section{Wellness motivation theory}

The development of theory and practice focused on individual efforts to promote cardiovascular health must include recognition of both the motives guiding behavior in older adults, as well as how these motives are transformed into personal intentions and plans, action, and integration [38]. Wellness Motivation Theory provides a framework for understanding yoga practice promoting cardiovascular health in older adults as a purposeful process of personal growth, integration of experience, and connection with others, reflecting meaning and potential, and resulting in being well and living values [39]. Within WMT, action is motivated not to address deficits in chronic illness, but in alignment with meaning; to foster potential as defined by the individual $[23,40,41]$. The WMT links the present, past, and future through self-knowledge and selfregulation processes guiding preparing for action, action, and the integration of action into life patterns.

As a guide for intervention research, Fleury \& Sidani [20] call for theoretical specificity fostering: (a) accurate conceptualization of the problem targeted by the intervention and the population responsive to the intervention; (b) specification of the critical inputs that operationalize the intervention; (c) delineation of contextual factors that influence the implementation and outcomes of the intervention, including characteristics of the target population, interventionists, and setting or environment that moderate the effects of the intervention on outcomes; and (d) understanding of the mechanisms or processes of change yielding the expected or desired outcomes, including identification of specific mediators of change that can determine why an intervention was or was not successful. Attention to these elements is essential to understanding why, how, and under what conditions the effects of the Yoga for HEART intervention occur (Figure 2). 


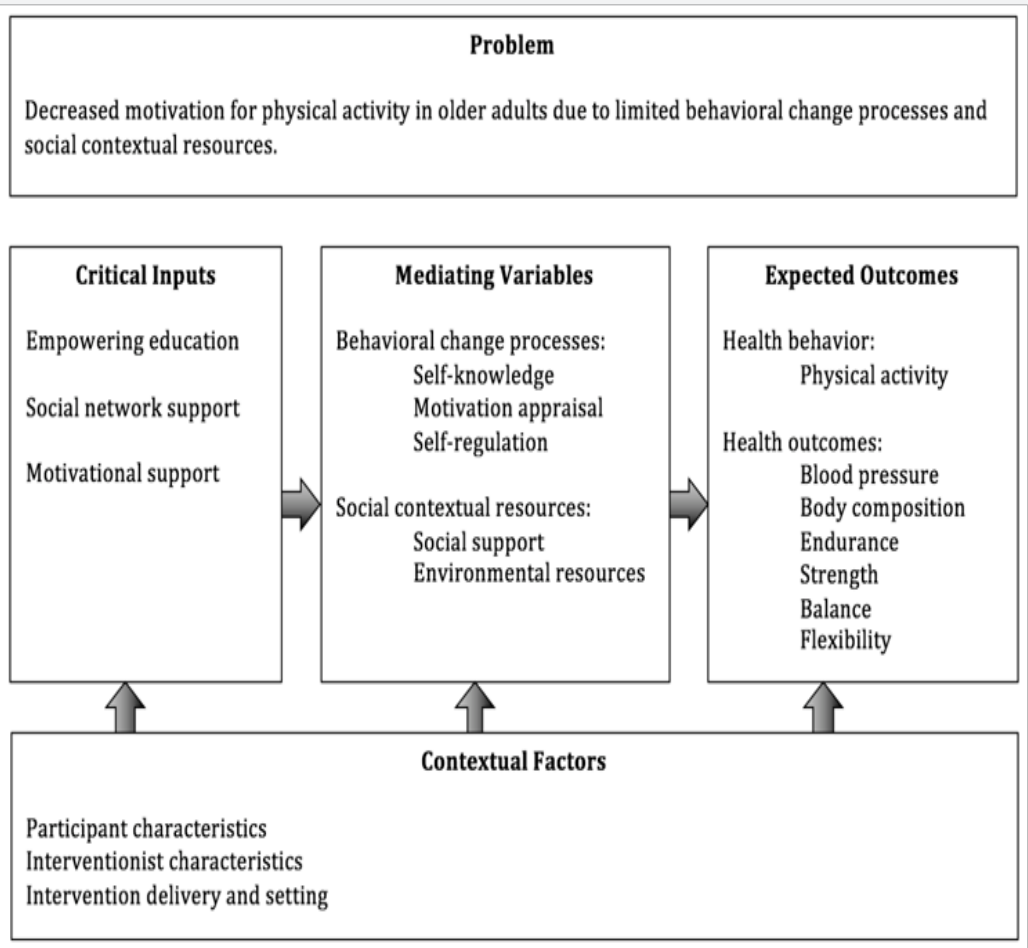

Figure 2: Theoretical components of the yoga for HEART intervention.

\section{Problem}

While yoga interventions have addressed the problem of CVD, physical inactivity, and associated health risks, few have focused on enhancing motivation for yoga practice to promote cardiovascular health in older adults. Motivation for health behavior is a complex phenomenon influenced by factors that evolve over time and across situations. A theorybased intervention that addresses the problem of decreased motivation will strengthen the ability to make explicit the nature and details of behavior change. As motivation is central to the initiation and maintenance of health behavior, it is essential for researchers and practitioners to develop and implement theorybased interventions that foster motivation for yoga practice and physical activity to improve cardiovascular health outcomes in older adults [39].

Yoga for HEART is focused on the processes of motivation and the patterning of individual health behavior in relation with the environment. Wellness Motivation Theory explains the problem of decreased motivation for health behavior change as limited social contextual resources and behavioral change processes [23,24]. Motivation for health behavior change in older adults is fostered by the identification and development of social contextual resources in interaction with behavioral change processes, including self-knowledge, motivation appraisal, self-regulation, social support, and environmental resources. Increasing awareness of and access to individual, social, cultural, community, and environmental resources may lead to favorable changes in physical activity and cardiovascular health among older adults.

\section{Critical inputs}

Critical inputs are the essential components or active ingredients of an intervention that are responsible for activating mechanisms of behavioral change, linking theoretical mediating processes to health behavior outcomes [22]. To meet the aims of enhancing motivation for physical activity and promoting cardiovascular health, the Yoga for HEART intervention is designed to operationalize key dimensions of WMT by increasing social contextual resources and behavioral change processes. On the basis of theoretical and empirical testing, the critical inputs of the Yoga for HEART intervention are intended to (a) deliver empowering education, (b) strengthen social networks, and (c) provide motivational support for health behavior change in older adults [42-44]. These critical inputs are designed to enhance behavioral change processes and social contextual resources, including self-knowledge, motivation appraisal, self-regulation, social support, and community resources.

\section{Empowering education}

Consistent with principles of Astanga yoga and Wellness Motivation Theory, the Yoga for HEART intervention will be delivered from a perspective of empowering education. Empowering education fosters the identification and generation of social contextual resources for health behavior change, advocating a participatory approach in which individuals are not 
passive recipients of knowledge, but the active subjects of their own learning experiences $[45,46]$. Individuals will be engaged in problem identification and critical thinking to analyze the social context of health concerns. The role of the interventionist is to serve as a resource, facilitating conditions for community members to share in dialogue and to partner in constructing intentions for individual and community change. Through the process of dialogue, participants will envision opportunities, develop strategies, and build resources to change their lives and communities [46].

In the Yoga for HEART intervention, participants will engage in empowering educational activities that foster the identification and development of personal, social, cultural, community, and environmental strengths and resources. Time and space will be provided during intervention sessions for participants to engage in dialogue and critically analyze the social context of culturally relevant health issues. Group discussions will include topics about (a) the cardiovascular and overall health benefits of yoga practice and other types of physical activity; (b) the advantages of progressing gradually and following an individualized plan; and (c) the use of strategies to maintain healthy patterns of physical activity and integrate behavioral change into daily life.

\section{Social network support}

Physical activity takes place in a social context of families, friends, and community settings that influence processes of behavioral change. Social network membership may provide a sense of purpose, promote recognition of self-worth, enhance motivation for self-care, and improve utilization of community resources [47]. Social network support is designed to provide social support and strengthen existing social networks [30]. The mobilization of naturally occurring social networks is essential to sustainability, as these networks are likely to endure following study implementation. Social network support promotes the capacity to identify and create social contextual resources for physical activity and health behavior change.

The Yoga for HEART intervention addresses key dimensions of social support, defined as aid or assistance exchanged through interpersonal relationships and interactions. Social network support will be operationalized through small group yoga sessions facilitated by the instructor. Participants are welcome to bring a friend or family member to yoga sessions to enhance social network support.

\section{Motivational support}

Motivational support refers to helping individuals establish realistic goals and enact behavioral strategies to accomplish these goals. It focuses on skill development to support the adoption and maintenance of behavioral change through: (a) exploration and verbalization of goals and values; (b) identification and development of personal and community resources; (c) anticipatory problem solving to overcome barriers; and (d) selfmonitoring and self-regulation of physical activity.
To provide motivational support, the Yoga for HEART intervention will assist participants in identifying and developing the knowledge, skills, strategies, and resources to overcome personal barriers and challenges to health behavior change. Time and space will be provided to facilitate group discussion related to personal goals and accomplishments, barriers and challenges related to physical activity, and problem-solving strategies to sustain motivation for behavioral change. Plans for the future will be shared and achievements will be celebrated throughout the intervention sessions.

\section{Contextual factors}

Contextual factors are elements that influence the implementation, mediators, and outcomes of an intervention, including the characteristics of participants receiving the intervention, the characteristics of the interventionist delivering the intervention, and the environmental setting or context in which the intervention is provided.

\section{Participant characteristics}

Participant characteristics consist of an individual's personal profile, including their health status and past experience with physical activity and/or yoga. Participant characteristics also include their level of social support and available resources. These characteristics can influence the implementation of the intervention, engagement in psychosocial processes of change, enactment of physical activity behavior, and intervention outcomes. The Yoga for HEART intervention will use a comprehensive screening and enrollment process to ensure that each individual is healthy enough to participate in physical activity. The Physical Activity Readiness Questionnaire+ (PAR-Q+) will be used to screen for health conditions and refer participants to their physician for medical clearance. The Yoga for HEART intervention is designed to focus attention on individual characteristics such as social support and community resources, as identifying and building these characteristics are essential to the process of health behavior change in sedentary older adults.

\section{Interventionist characteristics}

As the interventionist is the medium through which the intervention is delivered, the critical inputs of the intervention are provided through interactions between the interventionist and participants. As a result, the personal and professional characteristics of the interventionist play a role in the implementation and effectiveness of the intervention. Personal attributes include demographic characteristics (e.g., age, gender, and ethnicity), communication skills, and demeanor. The demeanor of the interventionist is important as it contributes to the establishment of trust and rapport, which form the basis of the relationship between the interventionist and participants. Professional characteristics include the educational background, experience, and competence of the interventionist. The Yoga for HEART intervention will be delivered by the PI, a registered nurse and a certified yoga instructor who has experience working with 
older adults. The interventionist will convey a warm, friendly, and nonjudgmental attitude when interacting with participants, and will communicate information clearly using verbal and written cues to promote understanding. Personal thank-you cards will be mailed to all participants to express gratitude for their participation in the Yoga for HEART intervention study.

\section{Delivery setting}

The context of an intervention is the environmental setting in which it takes place. The environmental context should have the necessary features and resources to facilitate the delivery of the intervention and achieve intended outcomes. The Yoga for HEART intervention will take place in a community-based yoga studio with ample space, adequate lighting and ventilation, and a quiet atmosphere to promote relaxation and meditation. Free parking, public transportation, and private restrooms are available at this location. An elevator provides access to the second floor of the building where the studio is located.

\section{Theoretical mechanisms}

Theoretical mechanisms are the intervening variables that are proposed to mediate behavioral change [48]. Theorybased interventions should specify how critical inputs are linked to mechanisms of behavioral change and explain the processes through which interventions achieve expected outcomes. A systematic review of yoga interventions to promote cardiovascular health in older adults found that theory-based yoga intervention research is sparse in the published literature [7]. The Yoga for HEART intervention is designed to address this gap, as WMT operationalizes and measures relevant theoretical constructs as a basis for testing mechanisms of behavioral change.

The intended effects of the Yoga for HEART intervention are favorable changes in physical activity behavior and cardiovascular health. Achievement of intervention outcomes is contingent upon increased awareness and use of social contextual resources, engagement in behavioral change processes, and participation in physical activity relative to baseline values. For significant improvements in health behavior and health outcomes to occur, participants must experience favorable changes in self-knowledge, motivation appraisal, self-regulation, social support, and community resources. The mechanisms responsible for change are contingent upon participation in Yoga for HEALTH intervention sessions and the fidelity of the intervention, including interventionist delivery and participant receipt [49].

\section{Attention control condition}

Participants randomly assigned to the attention control group will receive mailed weekly newsletters focused on aspects of healthy aging. Newsletters are adapted from materials developed by the National Institute on Aging [50] and presented in Table 3. Attention control group content will not be linked to wellness motivation theoretical constructs. Measurement times and testing procedures will be analogous for intervention and control group participants.

Table 3: Weekly healthy aging topics.

\begin{tabular}{|c|c|}
\hline Week & Topic \\
\hline 1 & Healthy eating after 50 \\
\hline 2 & A good night's sleep \\
\hline 3 & Aging and your eyes \\
\hline 4 & Dietary supplements \\
\hline 5 & Older drivers \\
\hline 6 & Flu -get the shot \\
\hline 7 & Beware of health scams \\
\hline 8 & High blood pressure \\
\hline 9 & Arthritis advice \\
\hline 10 & Forgetfulness: knowing when to ask for help \\
\hline 11 & Online health information: can you trust it? \\
\hline 12 & Medicines: use them safely \\
\hline
\end{tabular}

\section{Outcomes}

Variables categorized as intervention acceptability, demand, fidelity, and efficacy will be measured using standardized questionnaires and procedures upon participant enrollment (T1) and immediately post-intervention at 12 weeks (T2).

\section{Intervention acceptability}

Intervention acceptability is a reflection of participant expectations, preferences, and perceptions. The extent to which participants find an intervention acceptable determines the extent to which it may be appropriate for future full-scale testing. An intervention acceptability questionnaire administered to the intervention group at T2 will evaluate two key aspects of acceptability: (a) intervention components, including utility, effectiveness, credibility, and satisfaction; and (b) intervention delivery, including dose and format. Participants will be surveyed regarding the extent to which they agree with items representing each aspect of the intervention, using a scale ranging from one (strongly disagree) to five (strongly agree). They will also be encouraged to describe how the intervention may be improved, providing narrative data as an additional evaluation source.

\section{Intervention demand}

Understanding the factors that influence attendance and attrition (intervention demand) contributes to the refinement of future intervention studies. An attendance log will serve as a class roster for each group session to track participation over time. Weekly attendance rosters will be reviewed and phone calls will be placed to any missing participants. An attrition log will record when and why participants may leave the study, allowing the research team to evaluate acceptable and unacceptable intervention attributes. Participants who leave the study will be contacted via telephone and asked to share reasons for dropout. Attrition data will be compared between intervention and attention control groups. 


\section{Implementation fidelity}

Quality monitoring will be used to address implementation fidelity, or the extent to which group intervention sessions are delivered as planned. Implementation fidelity will be evaluated using field notes and an index of procedural consistency. Field notes will be documented and reviewed for each intervention session, outlining delivery methods, use of resources, tasks accomplished, and time spent on each task. An index of procedural consistency will be used to evaluate the degree of implementation and the frequency and duration of activities as a basis for quantifying intervention fidelity. The index of procedural consistency is designed to measure fidelity to intervention session objectives using a scale ranging from one (not at all delivered) to three (delivered very well).

\section{Mediating variables}

The Yoga for HEART intervention targets change in theoretical mediating variables to effect cardiovascular health outcomes. Theoretical mediating variables include behavioral change processes (i.e., self-knowledge, motivation appraisal, and self-regulation) and social contextual resources (i.e., social support and community resources). Each mediating variable will be measured at $\mathrm{T} 1$ and $\mathrm{T} 2$.

Self-knowledge provides a conceptual foundation for assigning meaning to the process of health behavior change [41]. Self-knowledge frames and guides the generation of goals, creation of intentions, and perception of individual capabilities $[23,24,51,52]$. It provides a context of meaning through which individuals acknowledge their hopes and fears for the future, facilitating the activation of needed processes to achieve health goals [41]. Self-knowledge contains detailed constructions of behavioral plans to realize and avoid desirable and undesirable future health outcomes. The content and function of selfknowledge are essential links between motivation and behavior. Accessing and building self-knowledge leads to understanding the motivating factors or reasons for planning and integrating physical activity into daily life. Self-knowledge will be assessed with the Possible Selves Questionnaire (PSQ), a set of four openended questions and three Likert-type items designed to elicit hoped-for and feared possible selves and activities to achieve or avoid these selves. Responses to open-ended questions will be systematically evaluated using qualitative content analysis. Inter-rater reliability for the PSQ ranges from $87 \%$ to $98 \%$ [53]. Self-knowledge will also be assessed with goal attainment scaling, an individualized approach to the measurement of personally meaningful goals and outcomes, evaluating the extent to which individual goals are achieved on a 5 -point scale $[54,55]$.

Motivation appraisal reflects personal readiness to initiate and maintain health behavior change [56]. Motivation appraisal is a process of intention formation and goal-directed behavior consistent with personal values, strengths, and resources. It provides an individually constructed map or model outlining expectations for behavior. Through motivation appraisal, individuals assess their goals, make judgments about the means best suited to attain those goals, and determine commitment to valued outcomes [57]. The creation of and commitment to a plan of action can activate self-relevant information and resources to mobilize goal-directed behavior such as physical activity [58]. Motivation appraisal will be measured using the Index of Readiness (IR). The IR contains nine items on a scale ranging from 1 to 5 (strongly disagree to strongly agree), with three subscales measuring reevaluation of lifestyle, barrier identification, and goal commitment. Total scale internal consistency has been reported at 0.89 [59].

Self-regulation reflects the cognitive, affective, and behavioral mechanisms through which individuals attempt to make strategies for health behavior change congruent with valued goals [60]. In self-regulation, intentions are transformed into personalized action. The pursuit and attainment of goals and the maintenance of self-determined standards for behavior are critical sources of motivation that involve self-regulation. Self-regulatory mechanisms are key to understanding volitional aspects of behavioral change. They reflect the ways in which individuals attempt to behave in accordance with their personal goals, even when goals conflict or change over time [56]. Selfregulation in maintenance of physical activity will be measured using the Index of Self-Regulation (ISR). The ISR contains nine items on a scale ranging from one to five (strongly disagree to strongly agree), with three subscales measuring reconditioning, stimulus control, and behavioral monitoring. Internal consistency estimates for the ISR have ranged from 0.70 to 0.90 , with a total scale re-test reliability of $0.82[60,61]$.

Social support from family and friends exerts a significant influence on health behavior. Members of the social network may encourage or limit individual participation in physical activity. Social contextual influences reflect the degree to which personal, social, and environmental resources are perceived as available on individual and community levels. Social support will be measured using the Social Support and Exercise Survey (SSES), a questionnaire that measures perceived support for physical activity from family and friends [62]. Internal consistency estimates for the SSES have range from 0.84 to 0.91 [63].

Community resources will be assessed using the Perceived Environmental Support Scale [63]. The Perceived Environmental Support Scale captures community resources for physical activity including walking trails and recreation centers. Testretest correlations have ranged from $r=0.68$ to $r=0.80$ for items pertaining to neighborhood environment and community resources, respectively [63]. Each questionnaire will be read verbally to participants by a research assistant trained in the data collection procedures.

Physical activity behavior will be measured at T1 and T2 using the ActiGraph GT3X+ accelerometer (ActiGraph, LLC, Pensacola, FL). Accelerometers are widely accepted by researchers as providing valid and reliable estimates of physical activity; the 
ActiGraph is the most commonly used brand of device [64- 66]. Total activity counts per day (TAC/d) will be calculated and daily time spent in sedentary, low, moderate, and vigorous intensity physical activity will be classified using established cut points [67-69].

Cardiovascular health outcomes including blood pressure, body mass index, and waist-hip ratio will be measured from T1 to T2. Blood pressure will be measured using an automated digital blood pressure cuff according to manufacturer recommendations. Methods will be consistent with Joint National Commission (JNC) guidelines [70]. Body mass index will be computed as weight in kilograms divided by height in meters squared. Weight will be measured to the nearest $0.1 \mathrm{~kg}$ using a digital scale. Height will be measured to the nearest $0.5 \mathrm{~cm}$ with a stadiometer, participant shoeless and standing erect. Body fat distribution will be determined by collecting three sets of waist and hip measurements to the nearest $\mathrm{cm}$ with a tape measure to calculate mean circumferences and compute waist-hip ratio.

Health-related physical fitness will be measured using the Senior Fitness Test (SFT) [71]. The SFT contains six items to assess endurance, strength, flexibility, and balance needed to perform common everyday activities safely and independently without undue fatigue. Each test item has demonstrated validity and reliability in community-based research studies with older adults, including the (a) 30-second chair-stand test; (b) 30-second arm curl test; (c) 6-minute walk test; (d) chair sitand-reach test; (e) back scratch test; and (f) 8-foot up-and-go test.

\section{Randomization}

Participants will be randomly assigned to the yoga intervention group or the attention control group immediately after providing informed consent, using a table of blocked random numbers generated from the Statistical Package for the Social Sciences (SPSS). Two blocks including lists of random treatment assignments for 20 individuals, assigned to two conditions (10 in each group) will be created. Random assignment enhances internal validity by maximizing the chance that participants in the intervention and control groups have similar characteristics and by minimizing the probability of systematic differences between groups. Participants will be informed of their group assignment, to the yoga intervention or the attention control group, with instructions regarding condition protocol and procedures.

\section{Statistical Methods}

This pilot intervention study uses a two-group, repeated measures, experimental design with an intention-to-treat (ITT) analysis, meaning that all participants who are randomized will be analyzed. Preliminary analysis will include descriptive statistics to characterize sample demographics and variable measures. Frequencies, means, and standard deviations will be computed on all measures. Data displays will be examined for normal distribution, outliers, and missing data, to be transformed prior to analysis as appropriate. Any missing data will be handled with list wise deletion in SPSS. The yoga intervention and attention control groups will be compared on all baseline variables using independent t-tests and/or chisquare tests. If significant differences are detected at baseline, analysis of covariance (ANCOVA) will be conducted. A repeated measures analysis of variance (ANOVA) will be conducted to evaluate the efficacy of the Yoga for HEART intervention in promoting behavioral change processes, building social contextual resources, increasing physical activity behavior, and improving cardiovascular health and physical fitness. Effect sizes will be calculated by subtracting attention control group means from intervention group means, divided by the pooled standard deviation. Descriptive statistics will be used to summarize participant feasibility and acceptability. Participants who drop out will be compared to those who complete the study on all baseline variables. Results may indicate potential sample bias and help determine generalizability of study findings to specific populations and settings.

\section{Discussion}

Cardiovascular disease is the leading cause of death in older adults. Yoga is a mind-body type of physical activity that has been shown to reduce the risk of CVD, yet few yoga interventions have focused on the process of health behavior change to promote cardiovascular health in older adults. The Yoga for HEART intervention is designed to increase motivation for physical activity and improve health outcomes in older adults with attention to the social contextual resources and behavioral change processes that support the initiation and maintenance of health behavior change in older adults. This pilot study will address gaps in the literature and advance our understanding of the mechanisms by which yoga promotes health by testing an innovative theory-based yoga intervention targeting older adults, a vulnerable and underserved population.

\section{Funding}

This work was supported with a pre-doctoral scholarship from the National Center Hartford Center of Gerontological Nursing Excellence at Arizona State University, College of Nursing and Health Innovation.

\section{References}

1. Benjamin EJ, Blaha MJ, Chiuve SE, Cushman M, Das SR, et al. (2017) Heart disease and stroke statistics-2017 update: A report from the American Heart Association. Circulation 135: 00-00.

2. World Health Organization (2017) Cardiovascular diseases.

3. Mozaffarian D, Benjamin EJ, Go AS, Arnett DK, Blaha MJ, et al. (2016) Heart disease and stroke statistics-2016 update: A report from the American Heart Association. Circulation 133(4): e38-e360.

4. He W, Goodkind D, Kowal P (2016) An aging world: 2015.

5. https://www.heart.org/idc/groups/ahamah

6. Ward BW, Clarke TC, Nugent CN, Schiller JS (2016) Early release of selected estimates based on data from the 2015 National Health Interview Survey. 
7. Barrows JL, Fleury J (2016) Systematic review of yoga interventions to promote cardiovascular health in older adults. West J Nurs Res 38(6): 753-781.

8. Iyengar BKS (2014) Yoga: The path to holistic health $\left(2^{\text {nd }} e d n\right)$. Dorling Kindersley, London.

9. Khalsa SBS, Cohen L, McCall T, Telles S (2016) The principles and practice of yoga in health care. Handspring Publishing, Edinburgh, UK.

10. Chu P, Gotink RA, Yeh GY, Goldie SJ, Hunink MGM (2016a) The effectiveness of yoga in modifying risk factors for cardiovascular disease and metabolic syndrome: A systematic review and metaanalysis of randomized controlled trials. Eur J Prev Cardiol 23(3): 291307

11. Cramer H, Lauche R, Haller H, Steckhan N, Michalsen A, et al. (2014) Effects of yoga on cardiovascular disease risk factors: A systematic review and meta-analysis. Int J Cardiol 173(2): 170-183.

12. Govindaraj R, Karmani S, Varambally S, Gangadhar BN (2016) Yoga and physical exercise-A review and comparison. Int Rev Psychiatry 28(3): 242-253.

13. Innes KE, Selfe TK (2016) Yoga for adults with type 2 diabetes: A systematic review of controlled trials. J Diabetes Res 2016: 6979370.

14. Li AW, Goldsmith CW (2012) The effects of yoga on anxiety and stress. Altern Med Rev 17(1): 21-35.

15. Pascoe MC, Bauer IE (2015) A systematic review of randomized controlled trials on the effects of yoga on stress measures and mood. J Psychiatr Res 68: 270-282.

16. Mooventhan A, Nivethitha L (2017) Evidence based effects of yoga practice on various health related problems of elderly people: A review. Journal of Bodywork and Movement Therapies.

17. Chu P, Pandya A, Salomon JA, Goldie SJ, Hunink MGM (2016b) Comparative effectiveness of personalized lifestyle management strategies for cardiovascular disease risk reduction. J Am Heart Assoc 5(3): e002737.

18. Patwardhan AR (2016) Developing a small theory of treatment of yoga. Journal of Yoga and Physiotherapy 1(2): 1-5.

19. US Department of Health and Human Services (2014) Social and behavioral theories.

20. Fleury J, Sidani S (2012) Using theory to guide intervention research. In: Melnyk BM, Morrison Beedy D (Eds.), Intervention research: Designing, conducting, analyzing, and funding. New York, USA, pp. 1136.

21. Prestwich A, Sniehotta FF, Whittington C, Dombrowski SU, Rogers L, et al. (2014) Does theory influence the effectiveness of health behavior interventions? Meta-analysis. Health Psychol 33(5): 465-474.

22. Sidani S, Braden CJ (2011) Design, evaluation, and translation of nursing interventions. West Sussex, Wiley-Blackwell, UK.

23. Fleury JD (1991) Empowering potential: A theory of wellness motivation. Nurs Res 40(5): 286-291.

24. Fleury J (1996) Wellness motivation theory: An exploration of theoretical relevance. Nurs Res 45(5): 277-283.

25. Bachman N (2011) The path of the yoga sutras: A practical guide to the core of yoga. Sounds True Publishing, USA.

26. Satchidananda SS (2012) The yoga sutras of Patanjali. VA: Integral Yoga Publications, Buckingham, England.

27. Boutron I, Altman DG, Moher D, Schulz KF, Ravaud P (2017) CONSORT statement for randomized trials of nonpharmacologic treatments: A 2017 update and a CONSORT extension for nonpharmacological trial abstracts. Annals of Internal Medicine 167: 40-47.
28. Eldridge SM, Chan CL, Campbell MJ, Bond CM, Hopewell S, et al. (2016) CONSORT 2010 statement: Extension to randomized pilot and feasibility trials. Pilot and Feasibility Studies 355: i5239.

29. Moher D, Hopewell S, Schulz KF, Montori V, Gotzsche PC, et al. (2010) CONSORT 2010 explanation and elaboration: Updated guidelines for reporting parallel group randomized trials. BMJ 340: c869.

30. Yeom H, Fleury J (2014) A motivational physical activity intervention for improving mobility in older Korean Americans. West J Nurs Res 36(6): 713-731.

31. Silva Smith A, Fleury J, Belyea M (2013) Effects of a physical activity and healthy eating intervention to reduce stroke risk factors in older adults. Prev Med 57(5): 708-711.

32. American College of Sports Medicine (2014) ACSM's guidelines for exercise prescription and testing $\left(9^{\text {th }}\right.$ edn), Lippincott Williams \& Wilkins, Philadelphia, USA.

33. Nelson ME, Rejeski WJ, Blair SN, Duncan PW, Judge JO, et al. (2007) Physical activity and public health in older adults: Recommendations from the American College of Sports Medicine and the American Heart Association. Circulation 116: 1094-1105.

34. Marcus BH, Forsythe LH (2009) Motivating people to be physically active. $\left(2^{\text {nd }} e d n\right)$, Human Kinetics, Champaign, IL, USA.

35. US Department of Health and Human Services (2008) 2008 physical activity guidelines for Americans.

36. Newkirk LA, Kim JM, Thompson JM, Tinklenberg JR, Yesavage JA, et al. (2004) Validation of a 26-point telephone version of the Mini-Mental State Examination. J Geriatr Psychiatry Neurol 17(2): 81-87.

37. Feuerstein G (2011) The path of yoga: An essential guide to its principles and practices. Shambhala Publications, Boston, USA.

38. McMahon S, Fleury J (2012) Wellness in older adults: A concept analysis. Nurs Forum 47(1): 39-51.

39. Baumeister RF (2016) Toward a general theory of motivation: Problems, challenges, opportunities, and the big picture. Motivation and Emotion 40(1): 1-10.

40. Fleury J, Kimbrell LC, Kruszewski MA (1995) Life after a cardiac event: Women's experience in healing. Heart Lung 24(6): 474-482.

41. Fleury J, Sedikides C (2007) Wellness motivation in cardiac rehabilitation: the role of self-knowledge in cardiovascular risk modification. Research in Nursing \& Health 30(4): 373-384.

42. Fleury J, Belyea M, Harrell J (2000) Physical activity among elderly African Americans: A test of the wellness motivation theory. Annals of Behavioral Medicine 22: 119 .

43. Fleury J, Keller C, Perez A (2009) Social support theoretical perspective. Geriatr Nurs 30(2): 11-14.

44. McMahon S, Vankipuram M, Fleury J (2013) Mobile computer application for promoting physical activity. J Gerontol Nurs 39(4): 1520.

45. Freire P (1970) Pedagogy of the oppressed. New York: Continuum, USA, pp. 1-58.

46. Minkler M, Wallerstein N (2008) Community-based participatory research for health: From process to outcomes ( $2^{\text {nd }}$ edn). Jossey-Bass, San Francisco, USA.

47. Perez A, Fleury J (2009) Wellness motivation theory in practice. Geriatr Nurs 30(2 Suppl): 15-20.

48. Abraham C, Michie S (2008) A taxonomy of behavior change techniques used in interventions. Health Psychol 27(3): 379-387.

49. Bellg AJ, Borrelli B, Resnick B, Hecht J, Minicucci DS, et al. (2004) Enhancing treatment fidelity in health behavior change studies: 
best practices and recommendations from the NIH behavior change consortium. Health Psychol 23(5): 443-451.

50. National Institute on Aging (2015) Health \& aging.

51. Bandura A (1989) Human agency in social cognitive theory. American Psychologist 44(9): 1175-1184.

52. Hooker K, Kaus CR (1992) Possible selves and health behaviors in later life. Journal of Aging and Health 4(3): 390-411.

53. Frazier LD, Hooker K, Johnson PM, Kaus CR (2000) Continuity and change in possible selves in a 5-year longitudinal study. Basic and Applied Social Psychology 22(3): 237-243.

54. Kiresuk TJ, Sherman RE (1968) Goal attainment scaling: A general method for evaluating comprehensive community mental health programs. Community Ment Health J 4(6): 443-453.

55. Turner Stokes L (2009) Goal attainment scaling (GAS) in rehabilitation: A practical guide. Clin Rehabil 23(4): 362-370.

56. Yeom HA, Choi M, Belyea M, Fleury J (2011) Psychometric evaluation of the index of self-regulation. West J Nurs Res 33(2): 268-285.

57. Fleury J, Cameron Go K (1997) Women's rehabilitation and recovery. Crit Care Nurs Clin North Am 9(4): 577-587.

58. Biggs J, Fleury J (1994) An exploration of perceived barriers to cardiovascular risk reduction. Cardiovasc Nurs 30(6): 41-46.

59. Fleury J (1994) The index of readiness: Development and psychometric analysis. J Nurs Meas 2(2): 143-154.

60. Fleury J (1998) The index of self-regulation: Development and psychometric analysis. J Nurs Meas 6(1): 3-17.

61. Moore SM, Charvat JM, Pashkow F, Ribisl P, Roberts BL, et al. (2006) Effects of CHANGE intervention to increase exercise following cardiac events. Ann Behav Med 31(1): 53-62.
62. Sallis JF, Grossman RM, Pinski RB, Patterson TL, Nader PR (1987) The development of scales to measures social support for diet and exercise behaviors. Prev Med 16(6): 825-836.

63. Sallis JF, Johnson MF, Calfas KJ, Caparosa S, Nichols JF (1997) Assessing perceived physical environmental variables that may influence physical activity. Res Q Exerc Sport 68(4): 345-351.

64. Bassett DR, Troiano RP, McClain JJ, Wolff DL (2014) Accelerometerbased physical activity: Total volume per day and standardized measures. Med Sci Sports Exerc 47(4): 833-838.

65. Cain KL, Conway TL, Adams MA, Husak LE, Sallis JF (2013) Comparison of older and newer generations of ActiGraph accelerometers with the normal filter and the low frequency extension. Int J Behav Nutr Phys Act 10: 51.

66. Cheung VH, Gray L, Karunanithi M (2011) Review of accelerometers for determining daily activity among elderly patients. Arch Phys Med Rehabil 92(6): 998-1014.

67. Aguilar Farías N, Brown WJ, Peeters GM (2014) ActiGraph GT3X+ cutpoints for identifying sedentary behaviour in older adults in free-living environments. J Sci Med Sport 17(3): 293-299.

68. Keadle SK, Shiroma EJ, Freedson PS, Lee IM (2014) Impact of accelerometer data processing decisions on the sample size, wear time and physical activity level of a large cohort study. BMC Public Health 14: 1210 .

69. Sasaki JE, John D, Freedson PS (2011) Validation and comparison of ActiGraph activity monitors. J Sci Med Sport 14(5): 411-416.

70.US Department of Health and Human Services (2003) The Seventh Report of the Joint National Committee on prevention, detection, evaluation, and treatment of high blood pressure.

71. Rikli RE, Jones CJ (2013) Senior fitness test manual. ( $2^{\text {nd }}$ edn), Human Kinetics, Champaign, IL, USA.

\section{Your next submission with Juniper Publishers will reach you the below assets}

- Quality Editorial service

- Swift Peer Review

- Reprints availability

- E-prints Service

- Manuscript Podcast for convenient understanding

- Global attainment for your research

- Manuscript accessibility in different formats

( Pdf, E-pub, Full Text, Audio)

- Unceasing customer service

Track the below URL for one-step submission https://juniperpublishers.com/online-submission.php 